\title{
Supplementary Information: Averting evolutionary suicide from the tragedy of the commons
}

\author{
J. Stephen Lansing, Ning Ning Chung, Guy S. Jacobs \& Lock Yue Chew
}

\begin{abstract}
Overview
Here we describe our use of principal components analysis (PCA), Fisher Information and energy landscape analysis as well as results relating to them not covered in the main text. We first detail our application of PCA in Section A. Next in Section B we explain our the projection of the average of the 19 descriptors of each subak to the three-dimensional PCA space formed by the first three principal components. We analyze the distribution of principal component values projected from the 19 subak's descriptors into the PCA space. We explore the evidence for multiple attractors based on the clustering of subaks in this embedding linear subspace. The evidence that more than one attractor exists in the survey data leads us to exploit the distribution of principal component values projected from the 19 subak's descriptors into the PCA space. We use the first two principal components to evaluate the Fisher Information (FI), which unlike PCA does not assume linearity of correlations. To calculate the FI we obtain the best-fit of the distributions of the first and second principal components of each subak to a set of known distributions. To obtain the best-fit to the empirical distributions, we compare the distributions based on just the Gaussian distribution, to the Gaussian, Rayleigh, and Pareto distributions (see Section C). The resulting two versions of Fisher Information landscapes are then compared to evaluate the consistency and robustness of the results (see Section D). In Section E we utilize a statistical physics approach based on the Ising model to construct an energy landscape, to probe the relationship between the attractors. Our innovation lies in the use of Principal Components Analysis (PCA) as the basis for constructing the energy landscape. Through the principle of maximum entropy, the derived Boltzmann distribution gives high energy value to states that are less visited and lower energy to more frequently visited states. The consequence is an energy landscape where deep wells correspond to densely populated states while shallow well contains the less populated states. PCA is used to cluster Subaks with similar social-ecological behaviour at different parts of the PCA space. We observe a large group of subaks with cooperative and stable social-ecological behaviour clustered together forming a deep attractor in the energy traditional behaviour. Only four subaks deviate from the cooperative norm, which is unsurprising given the persistence of the subak system in Bali. Using the methods summarized above we show that these four subaks need not be treated as outliers. Instead they help to define pathways towards or away from evolutionary suicide. In Section F we provide an English translation of all survey questions. Section G provides the MATLAB code for our analyses.
\end{abstract}

\section{A. Principal Component Analysis (PCA)}

A set of 35 questions that covers the social-ecological issues of the subaks was extracted from the 52 questions of the survey questionnaire given in section E. These questions constitute the 35 categorical descriptors (or variables) in our preliminary analysis (see Table S1). We define a data matrix $X$ of size $M \times N$ to capture the response of the farmers to these survey questions. While each row of $X$ relates to one of the 35 descriptors, each column of $X$ corresponds to a farmer out of a sample of 493 farmers from 20 subaks $^{1}$. Thus, $M=35$ and $N=493$. Specifically, the matrix element $X_{i j}$ represents the response of farmer $j$ to a question that is related to descriptor $i$.

To begin PCA, we form a zero-mean data matrix $Y$ with element

$$
Y_{i j}=X_{i j}-\bar{X}_{i}
$$

Note that

$$
\bar{X}_{i}=\frac{1}{N} \sum_{j=1}^{N} X_{i j}
$$

is the mean of descriptor $i$, which is obtained by averaging all the farmers' response for that descriptor. The $M \times M$ covariance matrix of descriptors $C$ is then determined as follows:

$$
C=\frac{1}{N} Y Y^{T}
$$

${ }^{1}$ For the sake of computation and ease of reference, we have placed all the farmers that belong to the same subak contiguously within a block in the matrix $X$. 
where the superscript $T$ indicates matrix transposition. Due to the different nature of each descriptor as exhibited by their different physical units, it is necessary to render them dimensionless. This is achieved through the process of normalization using $s_{i}=\sqrt{C_{i i}}$. In this respect, it is more appropriate to adopt the correlation matrix $R$ with elements

$$
R_{i j}=\frac{C_{i j}}{s_{i} s_{j}}
$$

in our analysis. In matrix form, we can also express $R=D C D$, where $D$ is a diagonal matrix with element $D_{i i}=1 / s_{i}$. Singularvalue decomposition performed on $R$ then leads to the following factorization:

$$
R=E \Lambda E^{T}
$$

where $\Lambda$ is a diagonal matrix of positive singular values such that $\Lambda_{i i}>\Lambda_{j j}$ for $i<j$; and $E$ is an orthogonal matrix with its $i$-th column being the loadings vector (or eigenvector) that corresponds to the $i$-th singular value (see Table S2 for the loading matrix of the 35 descriptors up to the third component). Our analysis at this stage has uncovered that the first and second principal component explain only $48.5 \%$ of the data. This can be improved by removing descriptors that are relatively insignificant and independent by means of higher-order clustering. The approach basically discriminates irrelevant descriptors from the more relevant ones through a distance matrix, as displayed in Fig. S1 via a cluster map of the correlation matrix. The consequence is a reduction in the number of descriptors from 35 to 19. These 19 descriptors are highlighted in red in Table S1: 4, 13, 14, 15, 17, 24, 27, 28, 25, 30, 31, 3, 18, 19, $35,22,23,33,34$. With these descriptors, we then form a reduced $19 \times 493$ data matrix $X$ and its associated $19 \times 19$ covariance matrix $C$. Our analysis based on these revised matrices has revealed that the first and second principal component now account for $62 \%$ of the data, justifying the use of the 19 descriptors as a basis for our subsequent detailed analysis.

Figure S1 reveals several interesting relationships between the descriptors. Descriptor 2 anti-correlates with descriptor 3. Farmers who inherited a farm usually do not sharecrop with other land owners. Exceptions are those in Betuas and Selukat who sold and leased their own land back, and so became sharecroppers. On the other hand, descriptors 24, 26 and 27 correlate with each other. These three descriptors, namely, fines, plan work and written rules followed are related to cooperativity of the farmers. Lastly, correlation between descriptors 17 and 18 implies that the farmers believe that they experience the same frequency of water shortage as other farmers in the Subak.

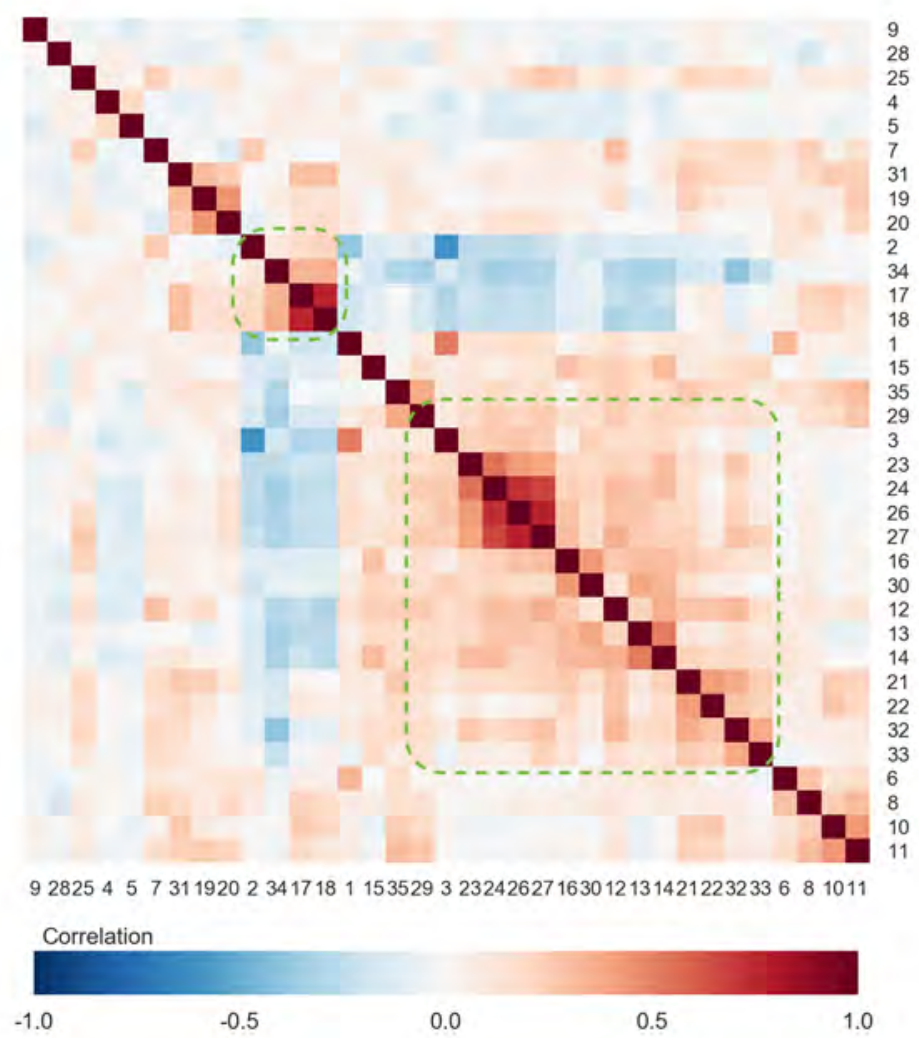

Figure S1: Cluster map for the correlation matrix of the 35 descriptors. The reduced set of the 19 descriptors are marked in dashed boxes. 
Table S1: A set of 35 questions were used in the preliminary analysis. A subset of 19 questions (in red) were used in the more detailed analysis, see discussion on higher-order clustering above.

\begin{tabular}{|c|c|c|}
\hline $\mathbf{S} / \mathbf{N}$ & Descriptor & Question Number \\
\hline 1 & Own farmland & Q4a \\
\hline 2 & Sharecrop land & Q4b \\
\hline 3 & Inherit a farm & Q5 \\
\hline 4 & Purchase & Q6 \\
\hline 5 & Sold farm & Q7 \\
\hline 6 & Income & Q8 \\
\hline 7 & Harvest & Q9 \\
\hline 8 & Satisfaction with harvest & Q10 \\
\hline 9 & Origin & Q11 \\
\hline 10 & Condition of canals & Q12 \\
\hline 11 & Condition of fields & Q13 \\
\hline 12 & Synchronize & Q16 \\
\hline 13 & Attendance at meetings & Q17 \\
\hline 14 & Participation in maintenance & Q18 \\
\hline 15 & Attendance at rituals & Q19 \\
\hline 16 & Accept subak decisions & Q20 \\
\hline 17 & Water shortages in subak & Q21 \\
\hline 18 & Water shortages myself & Q22 \\
\hline 19 & Pests damage in subak & Q23 \\
\hline 20 & Pests damage myself & Q24 \\
\hline 21 & Thefts of water & Q25 \\
\hline 22 & Conflicts among members & Q26 \\
\hline 23 & Choose of subak head & Q27 \\
\hline 24 & Fines & Q28 \\
\hline 25 & Crop schedule followed & Q29 \\
\hline 26 & Plan work & Q30 \\
\hline 27 & Written rules followed & Q31 \\
\hline 28 & Fines frequency & Q36 \\
\hline 29 & Condition of subak & Q40 \\
\hline 30 & Decisions of subak accepted & Q41 \\
\hline 31 & Technical problems & Q42 \\
\hline 32 & Social problems & Q43 \\
\hline 33 & Caste problems & Q44 \\
\hline 34 & Class problems & Q45 \\
\hline 35 & Resilience & Q46 \\
\hline
\end{tabular}


Table S2: Loading matrix of the 35 descriptors. The components give the weights for each original variable when calculating the corresponding principal components.

\begin{tabular}{|c|c|c|c|c|}
\hline \multirow[t]{2}{*}{ Descriptor \# } & \multirow[t]{2}{*}{ Descriptor } & \multicolumn{3}{|c|}{ Components } \\
\hline & & 1 & 2 & 3 \\
\hline 1 & Own farmland & -0.4930 & -0.1161 & 0.4865 \\
\hline 2 & Sharecrop land & 0.6296 & 0.3209 & -0.5053 \\
\hline 3 & Inherited a farm & -0.7115 & -0.3327 & 0.5201 \\
\hline 4 & Purchase & 0.2216 & 0.0526 & 0.0265 \\
\hline 5 & Sold a farm & 0.2735 & 0.0017 & -0.0189 \\
\hline 6 & Income & -0.1851 & 0.3504 & 0.1580 \\
\hline 7 & Harvest & -0.2601 & 0.3534 & -0.3868 \\
\hline 8 & Satisfaction with harvest & -0.2382 & 0.6202 & 0.0760 \\
\hline 9 & Origin & -0.0084 & -0.0012 & 0.1038 \\
\hline 10 & Condition of canals & -0.1302 & 0.7449 & 0.1549 \\
\hline 11 & Condition of fields & -0.2518 & 0.7793 & 0.1579 \\
\hline 12 & Synchronize & -0.9389 & 0.1414 & -0.2091 \\
\hline 13 & Attendance at meetings & -0.8265 & -0.2190 & -0.1760 \\
\hline 14 & Participation in maintenance & -0.9252 & -0.0490 & -0.1766 \\
\hline 15 & Attendance at ritual & -0.5329 & -0.0070 & -0.2428 \\
\hline 16 & Accept subak decisions & -0.7682 & 0.0671 & -0.2185 \\
\hline 17 & Water shortages in subak & 0.6380 & 0.8560 & 0.1018 \\
\hline 18 & Water shortages myself & 0.6551 & 0.8125 & 0.0974 \\
\hline 19 & Pest damage in subak & -0.1991 & 0.5954 & 0.2867 \\
\hline 20 & Pest damage myself & -0.2217 & 0.4477 & 0.4460 \\
\hline 21 & Thefts of water & -0.7236 & 0.5689 & -0.0680 \\
\hline 22 & Conflicts among members & -0.4882 & 0.5741 & -0.1757 \\
\hline 23 & Choice of subak head & -0.8821 & -0.1956 & 0.1095 \\
\hline 24 & Fines & -1.0714 & -0.2398 & 0.1908 \\
\hline 25 & Crop schedule followed & -0.3364 & 0.3418 & -0.1613 \\
\hline 26 & Plan work & -1.0748 & -0.2018 & 0.1773 \\
\hline 27 & Written rules followed & -1.1050 & -0.1107 & 0.0731 \\
\hline 28 & Fines frequency & 0.1338 & -0.1192 & 0.1728 \\
\hline 29 & Condition of subak & -0.7318 & 0.1815 & -0.0234 \\
\hline 30 & Decisions of subak accepted & -0.6874 & -0.0021 & -0.0017 \\
\hline 31 & Technical problems & -0.2622 & 0.7410 & 0.1641 \\
\hline 32 & Social problems & -0.7575 & 0.2759 & -0.2664 \\
\hline 33 & Caste problems & -0.4417 & 0.3095 & -0.3807 \\
\hline 34 & Class problems & 0.8724 & 0.1958 & 0.2075 \\
\hline 35 & Resilience & -0.6273 & 0.4174 & 0.0107 \\
\hline
\end{tabular}


Through PCA, we first observed clear association between the 19 descriptors in the biplot of Fig. 1 in the main text. We noticed three groups of closely correlated descriptors, which we term groups 1,2, and 3. Group 1 contains correlated descriptors which depend directly or indirectly on the cooperativity of the farmers. Group 2 is anti-correlated with group 1 and corresponds to defection. The relevant descriptors here are converse to cooperativity and are mainly associated with problems such as limited water availability at both the individual and subak level. The survey questions in Group 2 also include the proportion of owners versus sharecroppers, and whether class differences (likely to be correlated with land ownership) affect non-cooperative behavior. Group 3 is related to social disharmony and is observed to be uncorrelated with groups 1 and 2. It has descriptors in the social domain such as social conflicts, social problems, caste, and frequency of water theft. 


\section{B. The first three principal components}

The fact that the first three principal component can account for about $72 \%$ of the data variance implies that a three-dimensional PCA space would be sufficient to give a relatively good representation of the subak data. Let us now visualize the data representation of each of the 20 subaks in this reduced-dimensional space. For this purpose, we first perform an average of the survey responses of the $N_{s}$ farmers of a particular subak $s$ to yield its mean descriptor vector $\vec{\chi}^{s}$ (of size $19 \times 1$ ) with component $i$ :

$$
\chi_{i}^{s}=\frac{1}{N_{s}} \sum_{j=I_{s}}^{I_{s}+N_{s}-1} X_{i j} .
$$

$I_{s}$ is an index to the column of matrix $X$. It locates the first farmer of subak $s$, with $s \in\{1,2,3, \ldots, 20\}$. Essentially, we have $I_{1}=1$ and $I_{s+1}=I_{s}+N_{s}$. Next, we perform a projection of the $\vec{\chi}^{s}$ of each of the 20 subaks onto the first three dominant principal components as follows:

$$
\text { Principal component } i \text { of the } s \text {-th subak }=\left(\vec{\chi}^{s}\right)^{T} \vec{E}_{i},
$$

where $\vec{E}_{i}$ is the $i$-th column vector of the eigenvector matrix $E$ given in Eq. (5) with $i \in\{1,2,3\}$. The result is a plot of each subak as a point in a 3-dimensional PCA subspace as shown in Fig. S2, with the coordinates of each subak in this space given in Table S3. In this plot, we observe a major cluster of 16 subaks with four outliers - Bertuas, Selukat, Mantring and Kulub Atas. We have highlighted the four outliers as well as the subak Pakudui. 


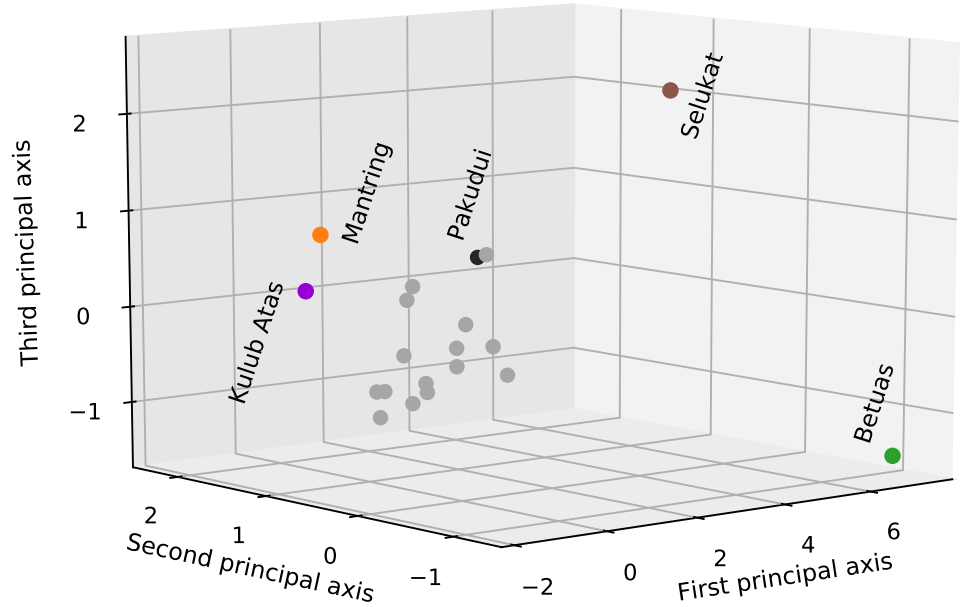

Figure S2: Clustering of the 20 subaks after projection into the three-dimensional principal component space.

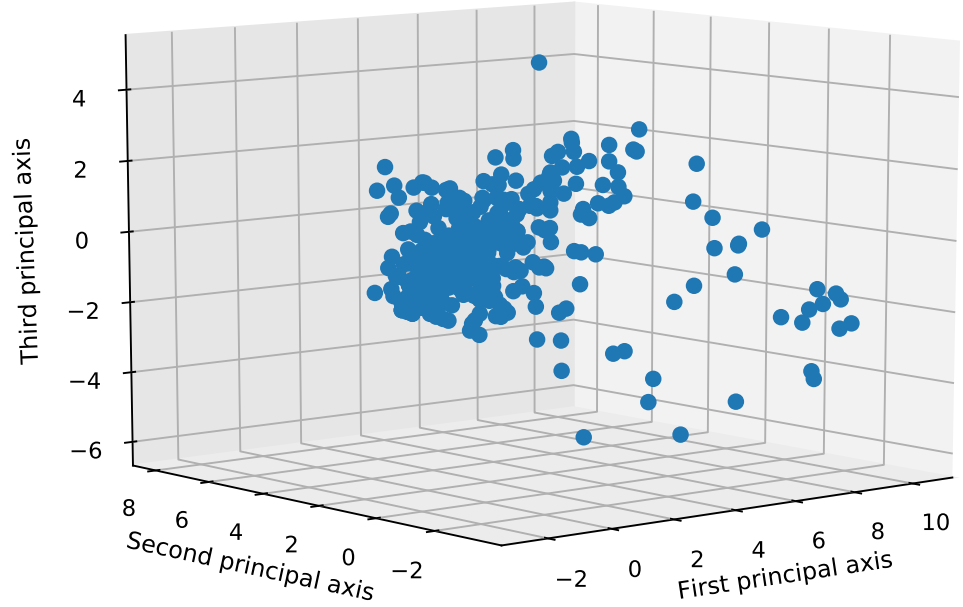

Figure S3: Projection of the 493 farmers into the three-dimensional principal component space. 
Table S3: Coordinates of subaks in PCA space

\begin{tabular}{llrrr}
\hline Subak \# & Subak & \multicolumn{3}{c}{ Coordinate } \\
& & PC1 & PC2 & PC3 \\
\hline & & & & \\
1 & Tampuaga hilir & -0.3074 & 0.3702 & 0.4461 \\
2 & Mantring & 1.4645 & 2.2680 & 0.6060 \\
3 & Tampuaga Hulu & -0.0055 & -0.2632 & 0.8482 \\
4 & Kebon & -1.6233 & 0.0730 & -0.7538 \\
5 & Calo & -0.8873 & 0.4751 & -0.6105 \\
6 & Cebok & -1.3418 & 0.1642 & -0.5231 \\
7 & Bayad & -1.3281 & -0.2643 & -0.3674 \\
8 & Timbul & -1.0915 & 0.0828 & -0.1632 \\
9 & Kedisan Kaja & -1.3188 & -0.5825 & -0.1452 \\
10 & Kedisan Kelod & -0.6860 & -0.6542 & 0.0311 \\
11 & Jasan & -0.6139 & 0.2817 & 0.3376 \\
12 & Selukat & 1.9215 & -1.2336 & 2.5161 \\
13 & Sebatu & -1.6206 & -0.2688 & -0.5508 \\
14 & Betuas & 7.3124 & -1.1603 & -1.4668 \\
15 & Pakudui & -0.2174 & -0.2720 & 0.8332 \\
16 & Aban & -0.6503 & -0.2588 & -0.0542 \\
17 & Teba & -0.3282 & -0.1996 & 0.1550 \\
18 & Dukuh & 1.1487 & 0.0663 & -0.4846 \\
19 & Tegan & -0.1011 & 0.3186 & -0.6336 \\
20 & Kulub Atas & 0.0572 & 1.7262 & 0.1665 \\
& & & & \\
\hline
\end{tabular}




\section{Least-square fitting of probability distribution}

PCA can be combined with Fisher Information (FI) to gain insight into attractor basins [1,2]. FI measures the amount of information that an observable random variable $X$ carries about an unknown parameter $\theta$ of a distribution that models $X$. It describes the probability that we will observe a given sample $X$, given a known value of $\theta$. FI is routinely used by astronomers to forecast what can be learned from future observations, with the aim of testing theoretical models while they are still in the design phase [3]. An advantage of FI is that it does not assume that correlations are linear. Instead it characterizes the probability distributions of each descriptor. The combination of PCA and Fisher Information produces a Fisher Information landscape, which gives a visual perspective of the regimes of stability of the dynamical system of interest. Intuitively, it is not surprising that most subaks are relatively robust (otherwise the subaks would be very vulnerable to evolutionary suicide), but that there are weaker attractors for component tragedies of the commons based on either social or environmental problems. FI supplements PCA with information about the topology of attractors in the survey data.

We investigate the diversity of survey responses in each subak by quantifying the Fisher information of its probability distribution of principal components. To proceed, we first project the responses of farmer $j$ in subak $s$ to the respective principal component as follows:

$$
\text { Principal component } i \text { of farmer } j \text { in the } s \text {-th subak }=\left(\vec{X}_{j}\right)^{T} \vec{E}_{i} \text {. }
$$

Note that $\vec{X}_{j}$ is the $j$-th column vector of the data matrix $X$ where $j \in\left\{j_{s}, j_{s}+1, \ldots, j_{s}+N_{s}-1\right\}$ such that the evaluation is performed solely on subak $s$. The probability distribution of principal component $i$ for subak $s$ is then easily obtained from a histogram of the set of principal component values computed from Eq. (8). We have yielded the probability distribution of principal component 1 and 2 for each of the 20 subaks based on this approach. In the following, we shall study these distributions with respect to two types of fitting. In the first type, the distribution is fitted solely to the Gaussian distribution. In the second type, a best-fit is to be achieved against one of the three statistical distributions: Gaussian, Rayleigh or Pareto.

The probability density function (pdf) of these three distributions is given by:

$$
p(x \mid \sigma)=\frac{1}{\sqrt{2 \pi \sigma^{2}}} \exp \left(-\frac{x^{2}}{2 \sigma^{2}}\right)
$$

for the Gaussian distribution;

$$
p(x \mid s)=\frac{x}{s^{2}} \exp \left(-x^{2} /\left(2 s^{2}\right)\right)
$$

for the Rayleigh distribution; and

$$
p(x \mid s, b)=\frac{b s^{b}}{x^{b+1}}
$$

for the Pareto distribution. The fitting is based on the criterion of lowest sum of squared error between the above distributions and the empirical distributions obtained from the histogram. The results of this analysis is displayed in Figs. S4 to S7. In general, we observe that Gaussian distribution has the best-fit to empirical distributions that are symmetrical. On the other hand, skewed distributions are better fitted to the Rayleigh distribution, while Pareto distribution is the best-fit for distributions that possess a sharp peak.

Next, we evaluate the Fisher information $F(\mu)$ of these fitted and best-fit distributions for both the principal component 1 and principal component 2 distribution where the parameter $\mu$ is the first statistical moment of the distribution. The evaluation is based on the following analytical form of Fisher information:

$$
\begin{gathered}
F\left(\mu_{\text {Gaussian }}\right)=\frac{1}{\sigma^{2}}, \\
F\left(\mu_{\text {Rayleigh }}\right)=\frac{2}{\pi} \int_{x}\left(\frac{x^{2}}{s^{3}}-\frac{2}{s}\right)^{2} p(x \mid s) d x
\end{gathered}
$$

and

$$
F\left(\mu_{\text {Pareto }}\right)=\frac{(b-1)^{2}}{b^{2} s^{2}}\left(3 b^{2}-3 b+1\right)
$$

which correspond to the Gaussian, Rayleigh and Pareto distribution respectively. Note that Eqs. (12) to (14) are derived by applying the definition of Fisher information:

$$
F(\theta)=\int_{x}\left(\frac{d L(\theta \mid x)}{d \theta}\right)^{2} p(x \mid \theta) d x
$$

to the respective pdfs given by Eqs. (9), (10) and (11), with $L(\theta \mid X)=\ln ((p(X \mid \theta))$ being the log-likelihood function. 

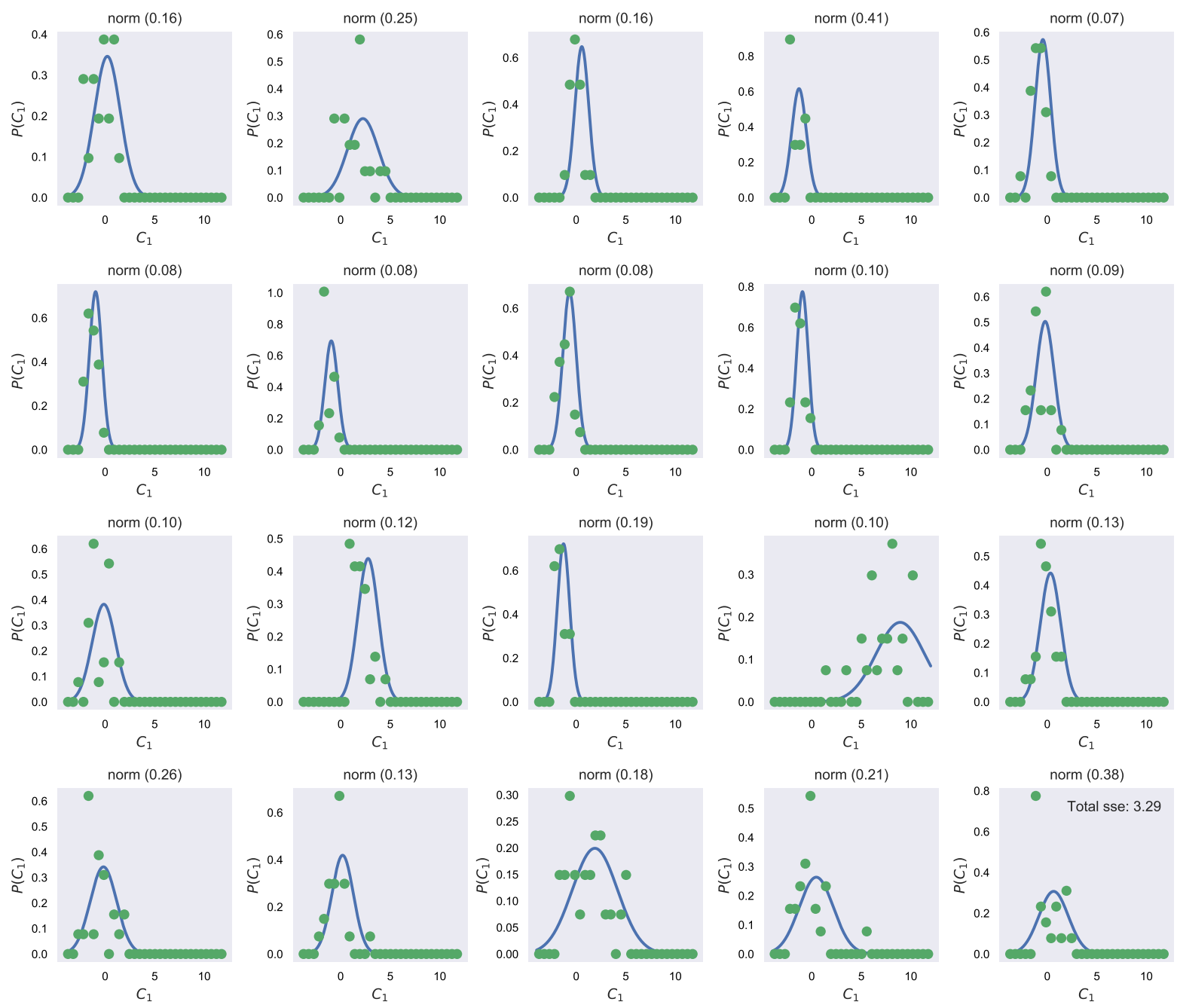

Figure S4: Least-square fitting of the probability distribution of the first principal component to the Gaussian distribution (marked as norm) for each subak. The fitted distribution is shown in the header for each subplot with the sum of squared error (sse) shown in the bracket. The total sse over the 20 subaks is shown in the last subplot. The fitting is used in the Fig. S8 (left panel) to form the Fisher Information Landscape by assuming that principal component 1 and 2 of all Subaks are Gaussian distributed. 

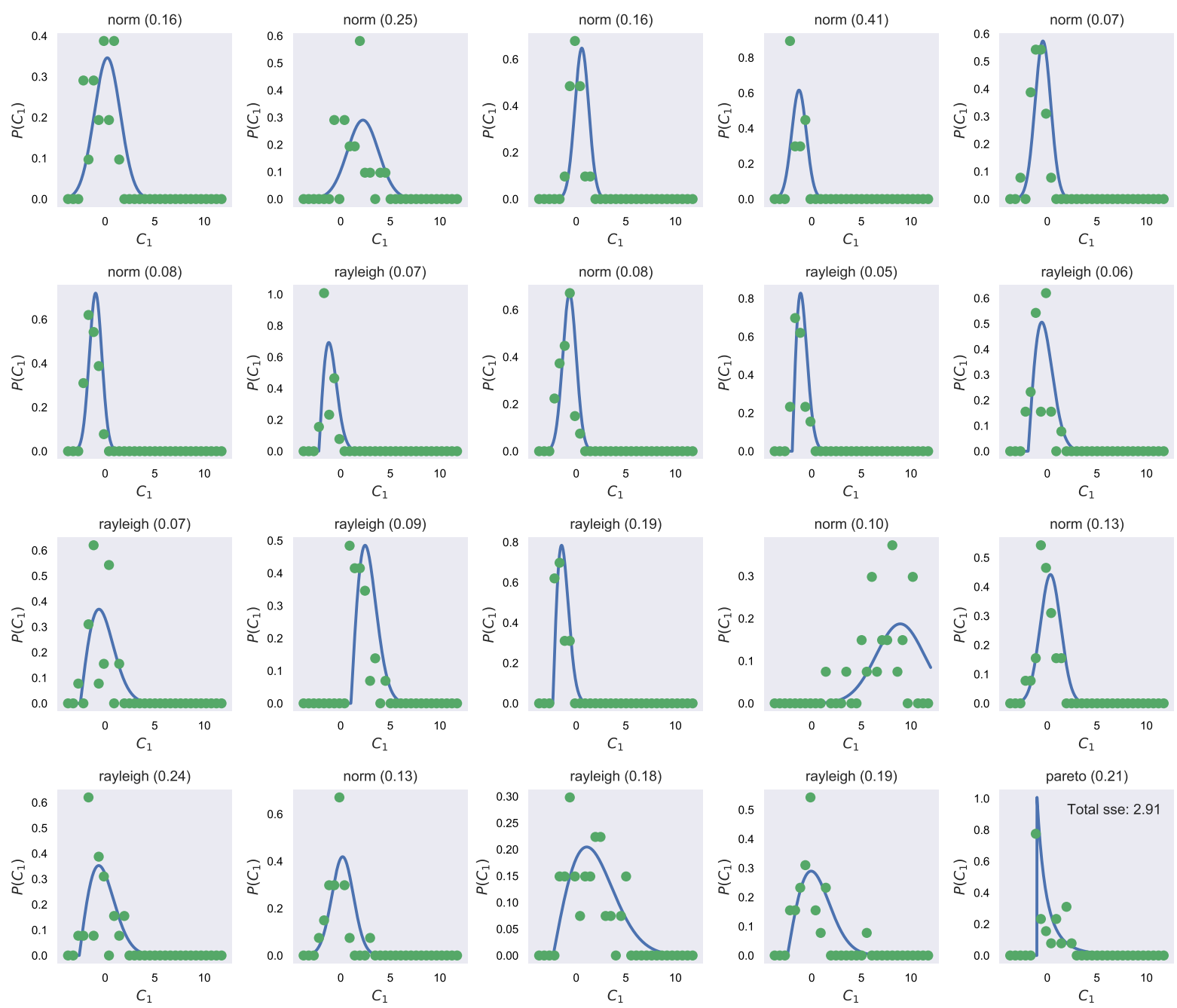

Figure S5: Best least-square fitting of the probability distribution of the first principal component to either the Gaussian, the Rayleigh, or the Pareto distribution (marked as norm, rayleigh and pareto respectively) for each subak. The fitted distribution is shown in the header for each subplot with the sum of squared error (sse) shown in the bracket. The total sse over the 20 subaks is shown in the last subplot. The fitting is used in the Fig. S8 (right panel) to form the Fisher Information Landscape. Compared to Fig. S4, Rayleigh distribution and Pareto distribution are indeed better fittings for the probability distribution of the first principal component for some of the Subaks. 

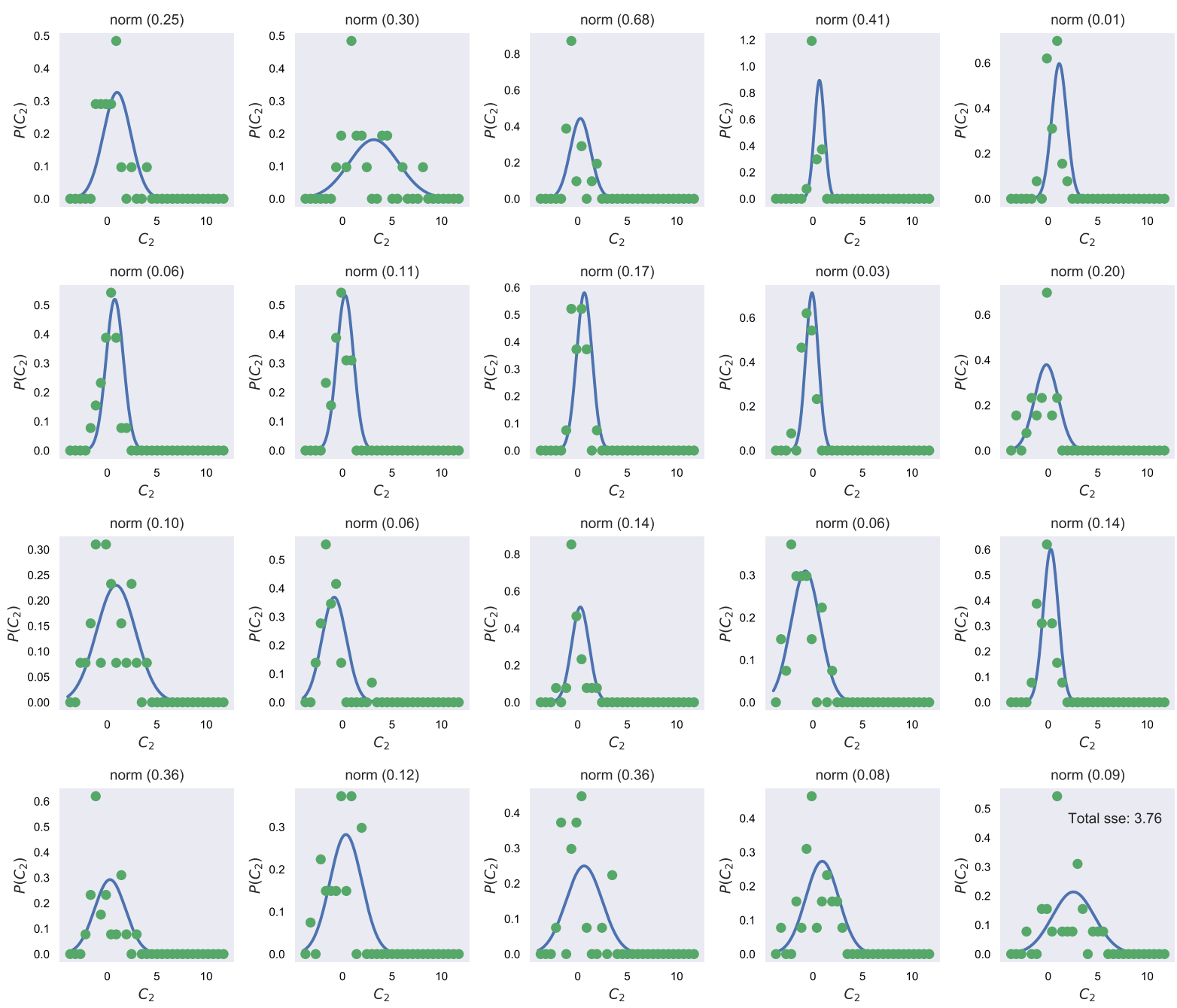

Figure S6: Least-square fitting of the probability distribution of the second principal component to the Gaussian distribution (marked as norm) for each subak. The fitted distribution is shown in the header for each subplot with the sum of squared error (sse) shown in the bracket. The total sse over the 20 subaks is shown in the last subplot. The fitting is used in the Fig. S8 (left panel) to form the Fisher Information Landscape by assuming that principal component 1 and 2 of all Subaks are Gaussian distributed. 

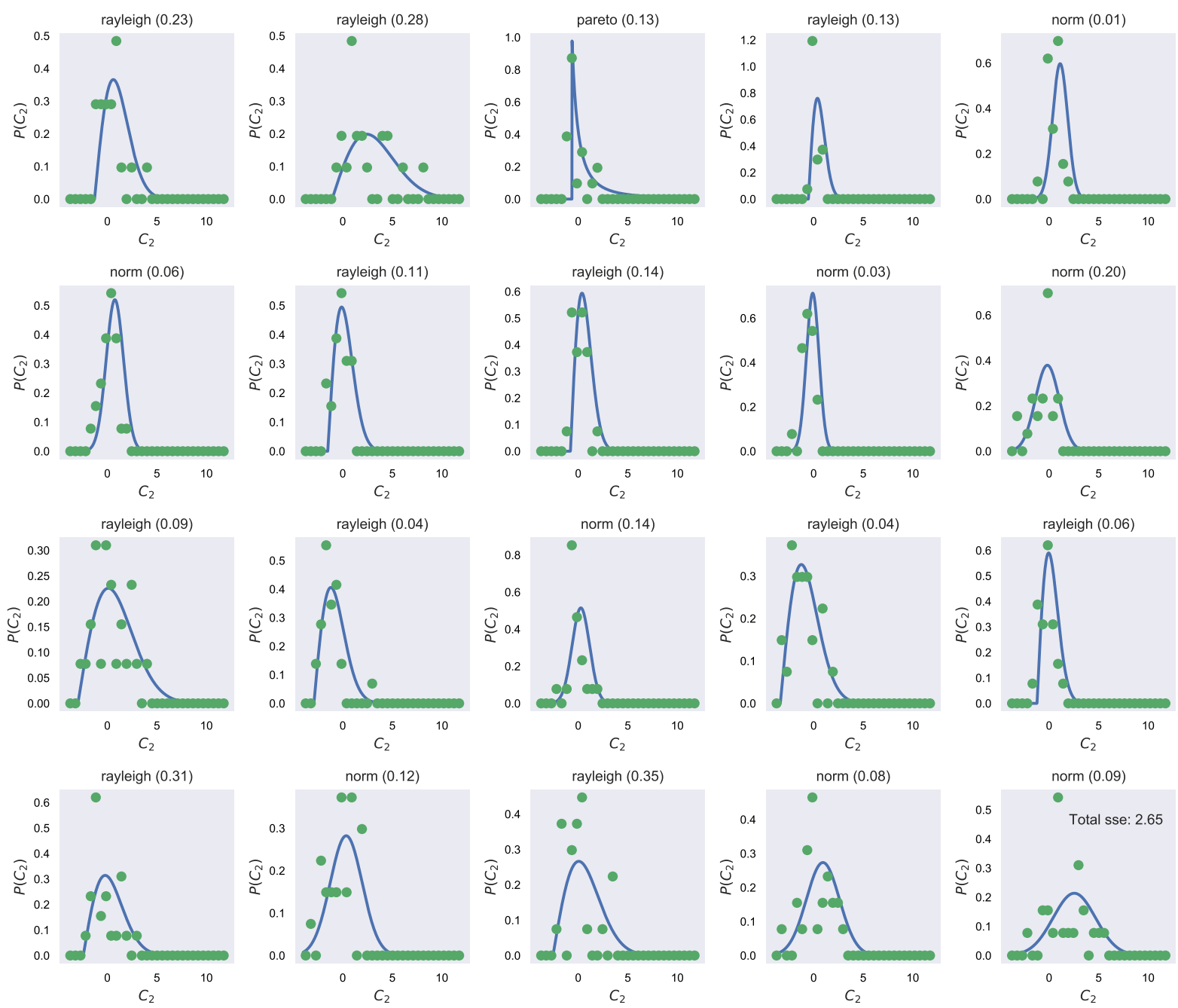

Figure S7: Best least-square fitting of the probability distribution of the second principal component to either the Gaussian, the Rayleigh or the Pareto distribution (marked as norm, rayleigh and pareto respectively) for each subak. The fitted distribution is shown in the header for each subplot with the sum of squared error (sse) shown in the bracket. The total sse over the 20 subaks is shown in the last subplot. The fitting is used in the Fig. S8 (right panel) to form the Fisher Information Landscape. 


\section{Comparison of Fisher Information Landscape.}

The analyses performed in sections B and C above allowed us to determine the Fisher Information Landscape of the system of 20 Balinese subaks. The Fisher Information Landscape is formed by the first two dominant principal component of the subak as its $x$ - and $y$-axes, with its associated Fisher information being the $z$-axis depicting the height of the landscape. Note that the former is computed from Eq. (7), while the latter results from a sum of the Fisher information obtained via the fitted probability distribution of principal component 1 and 2 of the subak.

We compare the Fisher Information Landscape between distributions of principal components best-fitted to the three distributions and those just-fitted to the Gaussian distribution. Except for minor variations, we observe that the main features of the attractor profiles are the same for the two versions. This indicates the robustness of the approach.
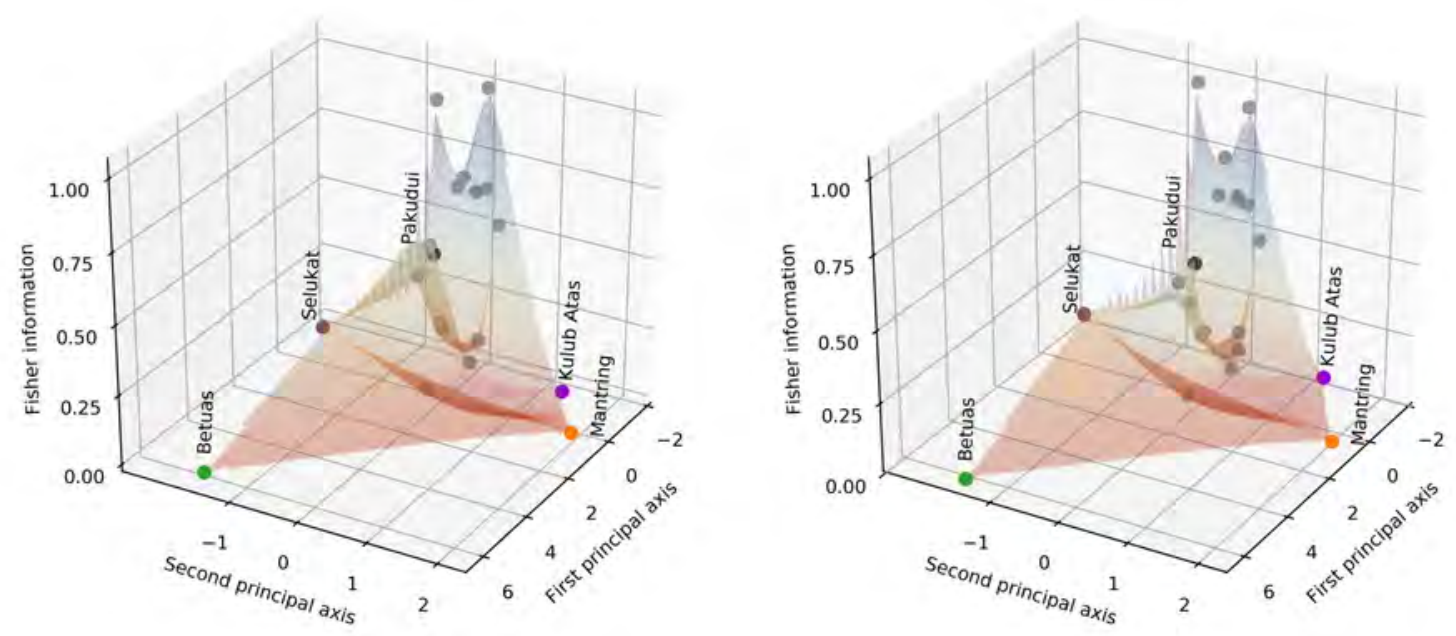

Figure S8: Comparison of Fisher Information Landscapes between versions just-fitted to the Gaussian distribution (left) and best-fitted to the three distributions (right).

\section{E. Energy landscape analysis}

The final step in the statistical analysis is to ask which combinations of variables would dominate the most probable transition paths between attractors in the Fisher Information Landscape. This approach, an energy landscape analysis, is borrowed from statistical physics. Because the variables are not physical or chemical but social-ecological, it does not offer a definitive prediction about actual transition paths. But it does show what the survey data alone tells us about which variables would dominate the simplest/likeliest transition, all other things being equal. Thus it can be regarded as suggesting hypotheses about change from the survey data.

As shown in Fig.8, attractor $\gamma$ appears as the steepest and deepest basin in the energy landscape. From this we calculate the variables that dominate the most probable path between attractors. Two contrasting paths appear. Social problems dominate the pathway from $\beta$ to $\gamma$, and are negligible for $\alpha$ to $\gamma$. These balance out for $\alpha$ to $\beta$. Water availability dominates the transition path from $\gamma$ to $\alpha$, because it is a constant problem for $\gamma$ subaks but essentially absent for $\alpha$. Attractor $\gamma$ appears as the only stable regime, the others are less well defined. 


\section{F. Translation of "Survei Subak 2012-13”, Steve Lansing October 2013}

Note: "Sawah" is wet rice terraced fields. Encoding of responses is indicated in square brackets following answer options. The descriptor that corresponds to each of the question is placed within bracket at the end of the question for each of the 35 descriptors.

1. Name of subak

2. Number of subak members

3. Where is your largest farm?
(a) same village where I live
(b) different village but same subdistrict as my residence
(c) different subdistrict, same district (kabupaten)

4. Amount of sawah you farm now:
(a) Owned by me hectares (own farmland)
(b) I sharecrop hectares (sharecrop land)

5. Have you inherited sawah? (inherit a farm)
(a) yes [1]
(b) no [0]

6. Have you purchased sawah? (purchase)
(a) yes [1]
(b) no $[0]$

7. Have you sold sawah? (sold farm)
(a) yes [1]
(b) no $[0]$

8. Your income from sawah, compared to your total family income, is: (income)
(a) $100 \%[1.0]$
(b) $66 \%[0.66]$
(c) $50 \%[0.5]$
(d) $25 \%[0.25]$
(e) $10 \%[0.1]$

9. Your last harvest of beras (unhulled rice) $\mathrm{kg} / \mathrm{ha}$ When? Which rice variety? (harvest)

10. Your level of satisfaction with this last harvest? (satisfaction with harvest)
(a) very satisfied [4]
(b) satisfied [3]
(c) less satisfied [2]
(d) not satisfied [1]

11. Were you born in this village? (origin)
(a) yes [1]
(b) no $[0]$

12. The condition of your subak's irrigation canals now, compared to the usual in Bali: (condition of canals)
(a) very good [4]
(b) $\operatorname{good}[3]$ 

(c) average [2]
(d) not good [1]

13. The condition of your subak's sawah now, compared to the usual in Bali: (condition of fields)
(a) very good [4]
(b) $\operatorname{good}[3]$
(c) average [2]
(d) not good [1]

14. Which is usually worse, irrigation water shortage or pest damage?
(a) water shortage
(b) pests

(these were not converted to a numerical scale)

15. Where is your sawah located?
(a) upstream sector of my subak
(b) mid section
(c) downstream sector of my subak
(these were not converted to a numerical scale)

16. How many subak members follow (a decision to) plant at the same time? (synchronize)
(a) all $100 \%[1.0]$
(b) most [0.85]
(c) half [0.5]
(d) less than half $[0.4]$

17. How many subak members (regularly) attend every subak meeting? (attendance at meetings)
(a) more than $80 \%[0.85]$
(b) between $60-80 \%[0.70]$
(c) less than $60 \%[0.5]$

18. How many subak members regularly participate in each collective work activity such as working on the canals? (participation in maintenance)
(a) more than $80 \%[0.85]$
(b) between $60-80 \%[0.70]$
(c) less than $60 \%[0.5]$

19. How many subak members regularly participate in religious activities carried out by the subak, like offerings? (attendance at rituals)
(a) more than $80 \%[0.85]$
(b) between $60-80 \%[0.70]$
(c) less than $60 \%[0.5]$

20. How many subak members usually follow (obey) the decisions of the subak head and the subak meetings? (accept subak decisions)
(a) all [1]
(b) most [0.75]
(c) half $[0.50]$ 
(d) less than half [0.25]

21. Are water shortages frequent in the subak during the dry season? (water shortages in subak)
(a) never [4]
(b) seldom [3]
(c) sometimes [2]
(d) frequent [1]

22. Do you yourself frequently experience water shortages? (water shortages myself)
(a) never [4]
(b) seldom [3]
(c) sometimes [2]
(d) frequent [1]

23. Are there frequent pest infestations? (pest damage in subak)
(a) never [4]
(b) seldom [3]
(c) sometimes [2]
(d) frequent [1]

24. Do you yourself frequently experience pests? (pest damage myself)
(a) never [4]
(b) seldom [3]
(c) sometimes [2]
(d) frequent [1]

25. Is water theft frequent in the subak? (theft of water)
(a) never [4]
(b) seldom [3]
(c) sometimes [2]
(d) frequent [1]

26. Are (social/personal) conflicts frequent among subak members? (conflict among members)
(a) never [4]
(b) seldom [3]
(c) sometimes [2]
(d) frequent [1]

Concerning decisions or results from subak meetings, which of the following reflect real democracy and which are just a democratic veneer?

27. Selection of subak head (choose of subak head)
(a) veneer of democracy [0]
(b) democratic/just (ie fair) [1]

28. Fines (fines)
(a) veneer of democracy [0]
(b) democratic/just (ie fair) [1] 
29. Choice of cropping pattern (crop schedule followed)
(a) veneer of democracy [0]
(b) democratic/just (ie fair) [1]

30. Organization of collective work (plan work)
(a) veneer of democracy [0]
(b) democratic/just (ie fair) [1]

31. Reading and following written rules of the subak (written rules followed)
(a) veneer of democracy [0]
(b) democratic/just (ie fair) [1]

32. If a farmer sees a person stealing water, what's the best thing to do?

(a) Ignore it and have faith in karmic consequences

(b) speak to the person

(c) report to the subak head

(d) bring it up in the subak meeting

(e) it depends.....

(these were not converted to a numerical scale)

33. Suppose a subak head collected money for irrigation repair, and after the work was done there was still money left unspent. The subak head kept some but not all of the money. If this became known, what would be the best response?
(a) Ignore it and have faith in karmic consequences
(b) speak to the person
(c) report to the subak head
(d) bring it up in the subak meeting
(e) it depends.....
(these were not converted to a numerical scale)

34. Who has the right to inherit sawah here?
(a) all sons equally
(b) the youngest son
(c) the oldest son
(d) other

(these were not converted to a numerical scale)

35. Who has the right to decide about imposing fines?
(a) Subak head
(b) Subak meeting
(c) Both
(d) neither has the right

(these were not converted to a numerical scale)

36. Are fines frequently imposed in your subak? (fine frequency)
(a) never [0.01]
(b) seldom [0.5]
(c) often [1] 
37. Are you married?
(a) Yes
(b) No

(these were not converted to a numerical scale)

If respondent is married:

38. Was your wife born in this community (this subak)?
(a) Yes
(b) No

(these were not converted to a numerical scale)

39. Is your wife a first or second cousin?
(a) Yes
(b) No

(these were not converted to a numerical scale)

40. The condition of your subak now is: (condition of subak)
(a) excellent, still intact [5]
(b) good enough [4]
(c) some problems have begun [3]
(d) not good [2]
(e) bad [1]

41. Are the results of subak meeting usually followed and carried out by subak members? (decisions of subak accepted)
(a) always [3]
(b) mostly [2]
(c) seldom [1]

42. Are there frequent technical problems in the subak, like water shortages, pests and low production? (technical problems)
(a) seldom [3]
(b) sometimes [2]
(c) frequently [1]

43. Are there frequent social problems in the subak, like conflict among members, water theft, failure to work together or to care about religious rites in the subak? (social problem)
(a) seldom [3]
(b) sometimes [2]
(c) frequently [1]

44. In your opinion, is there a connection between the strength/capability of the subak and caste conflicts within the subak? (caste problems)
(a) seldom [3]
(b) sometimes [2]
(c) frequently [1]

45. In your opinion, is there a connection between the strength/capability of the subak and differences in the level of prosperity or poverty of subak members? (Note: we refer to this as "class") (class problems) 

(a) seldom [3]
(b) sometimes [2]
(c) frequently [1]

46. The capability of the subak members to overcome difficulties, whether technical or social, is (resilience)
(a) Very capable [3]
(b) Capable [2]
(c) not very capable [1]

47. Imagine there are two candidates for subak head. Wayan really wants to be the head. Ketut will only serve if asked by the members. Who is the best choice?

(a) Wayan (if not selected, the subak could become hot)

(b) Ketut (because it is easy for the subak to believe in him)

(these were not converted to a numerical scale)

48. Who is best to become a subak head?
(a) a rich man
(b) an average man
(c) a poor man
(d) all are OK

(these were not converted to a numerical scale)

49. Who is best to become a subak head?
(a) a high caste man
(b) an ordinary caste man
(c) all are OK

(these were not converted to a numerical scale)

50. Are subak members embarrassed or fearful to be fined?
(a) embarrassed
(b) fearful
(c) neither

(these were not converted to a numerical scale)

51. Are all the members of the subak of ordinary caste?
(a) yes
(b) no, there are a few high caste men
(c) no, there are lots of high caste men
(these were not converted to a numerical scale)

52. What is (most) damaging to the subak?
(a) price of rice
(b) sawah converted to other uses
(c) local influences (other economic opportunities)
(d) functional capability of the subak (subak does not work well)

(these were not converted to a numerical scale) 


\section{G: Matlab codes}

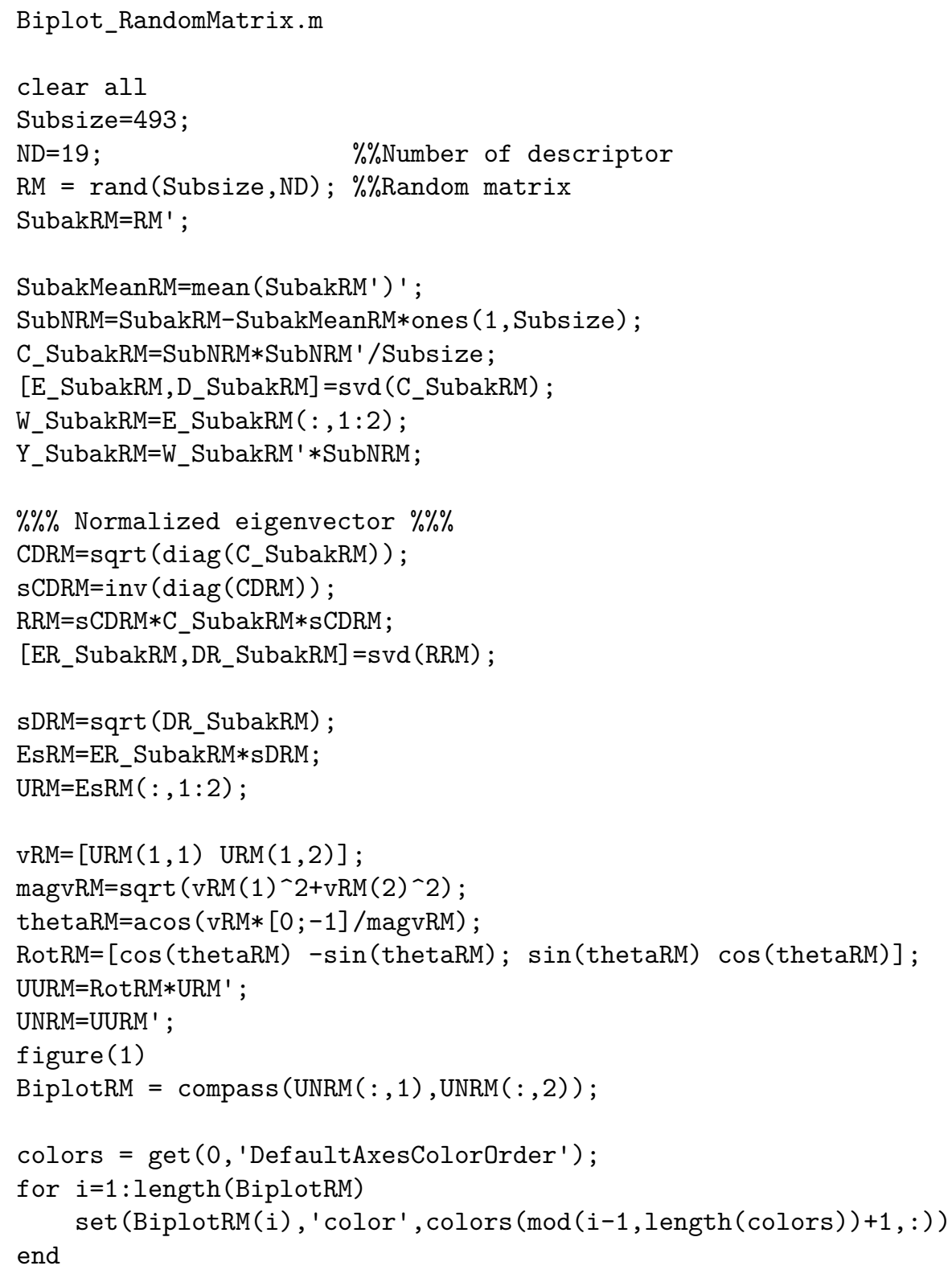




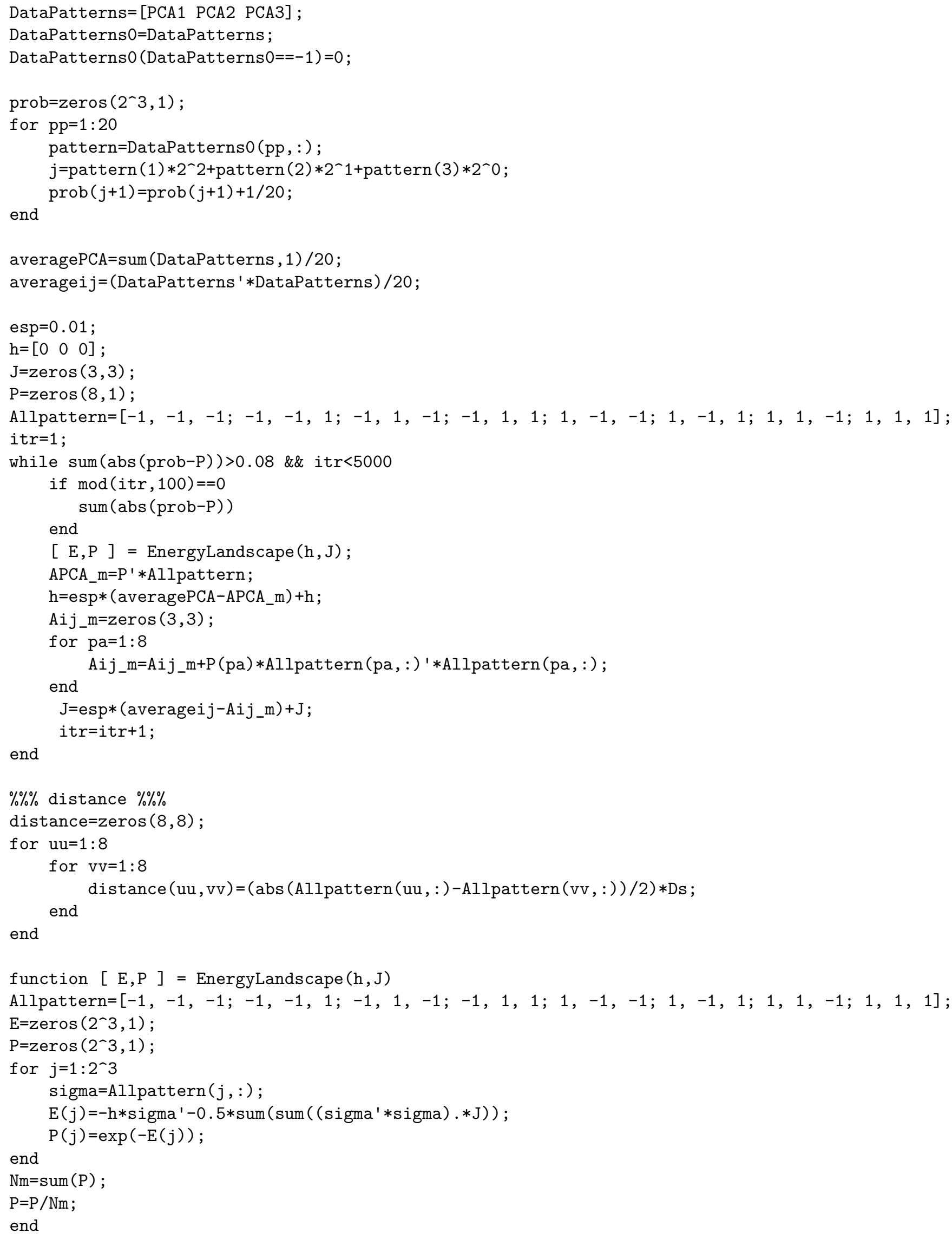




\section{Reference}

[1] Mayer, A. L., Pawlowski, C., Fath, B. D. \& Cabezas, H. Applications of Fisher Information to the Management of Sustainable Environmental Systems. (2007). In: Frieden, B. R. \& Gatenby, R. A. (eds) Exploratory Data Analysis Using Fisher Information. Springer, London

[2] Frank, S.A. Natural selection. V. How to read the fundamental equations of evolutionary change in terms of information theory. J. Evol. Biol. 25, 2377-2396 (2012).

[3] Machta, B. B., Chachra, R., Transtrum, M. K. \& Sethna, J. P. Parameter space compression underlies emergent theories and predictive models. Science 342, 604-7 (2013).

[4] Ezaki, T., Watanabe, T., Ohzeki, M. \& Masuda, N. Energy landscape analysis of neuroimaging data. Phil. Trans. R. Soc. A 375, 0287 (2017). 